\title{
Dor Articular na Osteoartropatia Hipertrófica Primária: Descrição de Caso e Revisão de Tratamento
}

\section{Arthralgia in Primary Hipertrophic Osteoarthropathy: Case Report and Review of the Treatment}

\author{
Aldo Lainetti ${ }^{(1)}$, Fernanda Satake Novaes ${ }^{(2)}$, Lívia Reis de Miranda ${ }^{(2)}$, Sílvio Henriques de Lira ${ }^{(3)}$
}

\section{RESUMO}

Apresentamos o caso de um paciente com baqueteamento digital e artrite que foi diagnosticado como tendo osteoartropatia hipertrófica primária. Essa é uma doença rara e benigna. Entretanto, artralgia e alterações cutâneas podem reduzir significativamente a qualidade de vida do paciente. Além de um breve resumo da doença, apresentamos o caso e a revisão da literatura, enfatizando o tratamento dessa condição para os clínicos em geral.

Palavras-chave: osteoartropatia hipertrófica primária, paquidermoperiostose, síndrome de Touraine-Solente-Golé, artralgia, baqueteamento digital.

\section{INTRODUÇÃO}

A osteoartropatia hipertrófica primária $(\mathrm{OHP})$ ou paquidermoperiostose é uma doença idiopática, familiar, benigna e autolimitada. A desordem parece ser herdada por um mecanismo de transmissão mendeliano ${ }^{(1)}$, que tem sido descrito como autossômico dominante, com penetrância e expressão variável na maioria das publicações ${ }^{(2-6)}$, ou como recessivo ${ }^{(1,6,7)}$ e que acomete principalmente homens $(90 \%)^{(1,3,6,6)}$.

Os sintomas podem ter início por volta de 1 ano de idade ou mais comumente na puberdade ${ }^{(8,9)}$ e tendem a estabilizar dentro de dez a vinte anos. Caracteriza-se por baqueteamento digital, neoformação óssea subperiosteal, principalmente das extremidades de ossos longos, acrosteólise, hipertrofia de tecidos moles e glândulas, principalmente em face e escalpo, seborréia, hiperidrose, pregueamento cutâneo na face e couro cabeludo (Cutis verticis gyrata), acne vulgar e outros sintomas menores ${ }^{(10)}$, como artrite e artralgia.

\begin{abstract}
We report the case of a patient with clubbing of the digits and arthritis who was diagnosed as having primary hipertrophic osteoarthropathy. This is a rare, benign disease. However, the arthralgia and skin changes can significantly impair the quality of life of the patient. In addition to a brief description of the disease, we present the case and a review of the literature regarding the treatment of this condition to the general practitioner.
\end{abstract}

Keywords: Primary hipertrophic osteoarthropathy, pachydermoperiostosis, Touraine-Solente-Golé syndrome, arthralgia, digital clubbing.

A forma completa da OHP apresenta todas as características mencionadas anteriormente; a forma incompleta apresenta as alterações ósseas sem Cutis verticis gyrata e a forma frustra é caracterizada pela presença de um ou mais achados cutâneos com mínimas ou sem anormalidades ósseas $^{(2,11-13)}$.

Há várias hipóteses para explicar as alterações encontradas na OHP, mas apenas o fator genético é confirmado. Parece que o fluxo sangüíneo periférico está aumentado no estágio inicial ativo da doença e reduzido na fase tardia desta, com parede arterial espessada e redução do lume, talvez em virtude do aumento do ativador de plasminogênio tecidual ${ }^{(11)}$.

Observam-se, ainda, aumento da proliferação fibroblástica na medula óssea e em biópsias de pele associadas à hiperplasia endotelial difusa, infiltrado linfo-histiocitário, além de adelgaçamento e agrupamento de fibras colágenas. Muitos estudos não puderam confirmar a diminuição do suprimento sangüíneo periférico proposto nos pacientes

Recebido em 08/03/07. Aprovado, após revisão, em 19/08/07. Declaramos a inexistência de conflitos de interesse.

Faculdade de Medicina de Valença (FMV), Rio de Janeiro.

1. Professor titular de Reumatologia da Faculdade de Medicina de Valença (FMV-RJ) e professor titular de Reumatologia (aposentado) da Faculdade de Medicina

da Universidade Federal Fluminense (UFF).

2. Graduanda da FMV.

3. Professor titular de Imagenologia da FMV

Endereço para correspondência: Lívia Reis de Miranda, Rua dos Mineiros, 136 - ap. 203, CEP 27600-000, Valença, RJ, e-mail: liviarmvalenca@yahoo.com.br 
com OHP ${ }^{(14)}$. Prostaglandinas E1, E2, F2, serotonina e bradicinina favorecem hipocratismo digital. Níveis elevados de osteocalcina na $\mathrm{OHP}$, associados à reabsorção óssea normal, favorecem a hipótese de que há desequilíbrio entre atividade osteoblástica aumentada e reabsorção óssea normal ${ }^{(11)}$.

Há evidências de níveis elevados de endotelina-1, $\beta$-tromboglobulina, fator de crescimento derivado de plaquetas (PDGF), fator de von Willebrand e fator de crescimento vascular endotelial (VEGF), tendo possível participação na progressão da doença e proliferação periosteal $^{(14)}$. Pacientes com OHP e aqueles com osteoartropatia associada a câncer de pulmão apresentam níveis elevados de VEGF(15).

Observou-se aumento da sensibilidade tecidual a hormônios sexuais circulantes em um paciente com OHP, o que induziria a maior produção e utilização tecidual do sistema de fator de crescimento epidérmico/fator de crescimento transformador $\alpha$. Esse sistema poderia, então, ser responsável pela hipertrofia tecidual na doença $a^{(2)}$. Sugeriu-se que o agente responsável pela OHP seria uma única substância, como um hormônio, com taxas anormais circulantes nos indivíduos afetados ${ }^{(14)}$.

O diagnóstico é essencialmente clínico, baseado na anamnese, no exame físico e na história familiar.

Os achados laboratoriais da OHP são similares aos da osteoartropatia hipertrófica secundária (OHS): a velocidade de hemossedimentação (VHS) pode estar elevada; testes urinários e sangüíneos, incluindo nível de complemento, são normais; fator reumatóide (FR) e anticorpos antinucleares (FAN) negativos. Quando há neoformação óssea considerável, os níveis de marcadores da remodelação óssea podem estar aumentados ${ }^{(2,3,16,17)}$. O líquido sinovial é caracteristicamente não-inflamatório, com predomínio de células mononucleares.

O diagnóstico diferencial entre OHP e OHS é praticamente impossível baseando-se apenas no exame físico. A diferenciação pode ser feita sabendo-se que na OHP o início é gradual na adolescência, há história familiar e não há causa base que a justifique, enquanto na OHS não há antecedentes familiares e ocorre remissão dramática com a retirada do fator causal, em geral, neoplásico ou distúrbio crônico $^{(4)}$ (Tabela 1).

Como a OHP gera aumento das partes moles das mãos e dos pés, durante muito tempo foi confundida com acromegalia, porém os níveis hormonais, a sela túrcica normal e a presença de baqueteamento digital afastam essa condição ${ }^{(4,7)}$. A OHP pode ser confundida com hanseníase, quando nela ocorre face leonina.
TABELA 1

Causas de osteoartropatia hipertrófica SECUNDÁRIa

\begin{tabular}{l} 
Doenças pulmonares \\
Neoplasias (carcinoma broncogênico) \\
Infecções (bronquite crônica, bronquiectasias) \\
Outras (fibrose cística, fibrose pulmonar, pneumoconiose, sarcoidose) \\
Doenças cardiovasculares \\
Endocardite bacteriana \\
Cardiopatias congênitas acianóticas \\
Ducto arterioso patente \\
Outras (aneurisma de aorta, prótese aórtica) \\
Doenças gastrointestinais \\
Neoplasias (carcinomas esofágico, hepático, cólon) \\
Infecções (disenteria amebiana) \\
Doenças hepatobiliares (cirroses biliar e hepática) \\
Outras (doença inflamatória intestinal, doença de Whipple) \\
Neoplasia (freqüentemente as com metástase intratorácica como linfomas) \\
Miscelânea (doenças do tecido conjuntivo, síndrome de POEMS) \\
Acropatia tireóidea \\
Síndrome da imunodeficiência adquirida (Aids) \\
\hline
\end{tabular}

Adaptado e modificado de Altman et al.(16).

Do ponto de vista radiológico, devemos fazer diagnóstico diferencial com doenças que produzem periostites simétricas, como leucemia, acromegalia, doença de Engelman, doença metastática e osteomielite crônica ${ }^{(4)}$. Deve-se fazer diferenciação também com artrite reumatóide e doença de Paget. Outros distúrbios associados com acrosteólise merecem participar do diagnóstico diferencial.

\section{RELATO DE CASO}

S.S.L.S., 23 anos, sexo masculino, compareceu em nosso Ambulatório de Reumatologia, no Hospital Escola Luiz Gioseffi Jannuzzi da Faculdade de Medicina de Valença (FMV), com queixa de dor e edema em tornozelos, joelhos e punhos associados a hiperemia e aumento de temperatura local havia cerca de um ano. O quadro articular foi progressivo (na ordem: tornozelo, joelhos e punhos), cumulativo e simétrico.

$\mathrm{Na}$ anamnese, relatou que a dor piorava aos movimentos e às baixas temperaturas, além de apresentar rigidez durante todo o dia. Notou-se aumento de volume dos dedos desde a adolescência. Ao ser questionado sobre o uso de medicamentos, revelou o uso prévio de indometacina com melhora do quadro álgico.

Em relação aos hábitos, é importante ressaltar o tabagismo (10 maços/ano) e o alcoolismo, sendo este último responsável por exacerbação do quadro clínico. 
Ao exame físico, constataram-se artrite de punhos, joelhos e tornozelos. O sinal da tecla estava presente em ambos os joelhos. O exame das mãos evidenciava baqueteamento digital que o paciente relatava ser familiar, unhas em vidro de relógio, além de espessamento da pele das mãos e punhos (Figuras l e 2). A pele, em geral, era espessada e oleosa, sendo o paciente portador de acne facial. Podia-se observar acentuada hiperidrose.

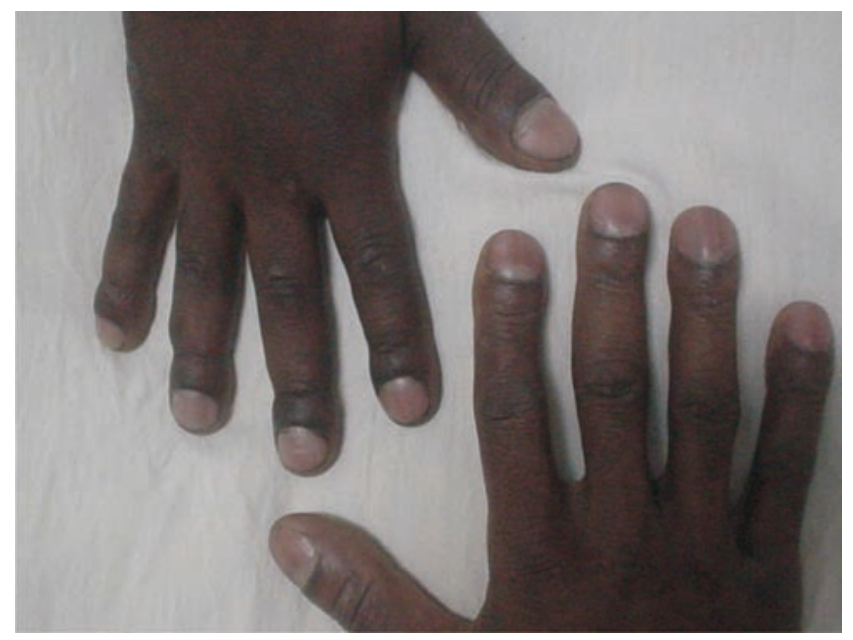

Figura 1 - Baqueteamento digital e espessamento de pele.

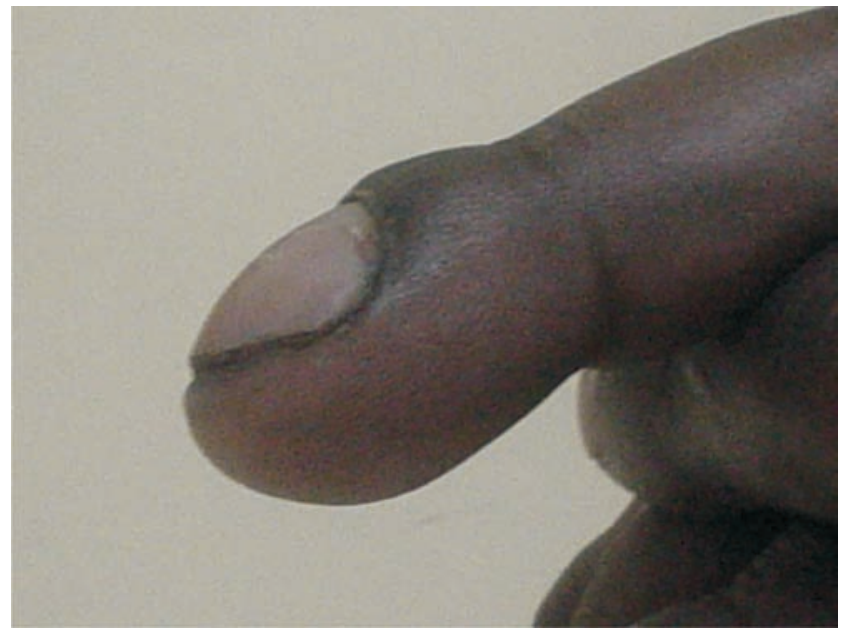

Figura 2 - Baqueteamento digital (visão lateral).

Solicitamos radiografias de tórax, punhos, mãos, joelhos e tornozelos, que foram analisadas por nossa equipe de radiologia. A radiografia de tórax não trazia nenhuma alteração, havia aumento de partes moles em todas as articulações acometidas e espessamento subperiosteal em extremidades dos ossos longos (Figura 3) que levaram o radiologista a aventar o diagnóstico de osteartropatia hipertrófica primária.

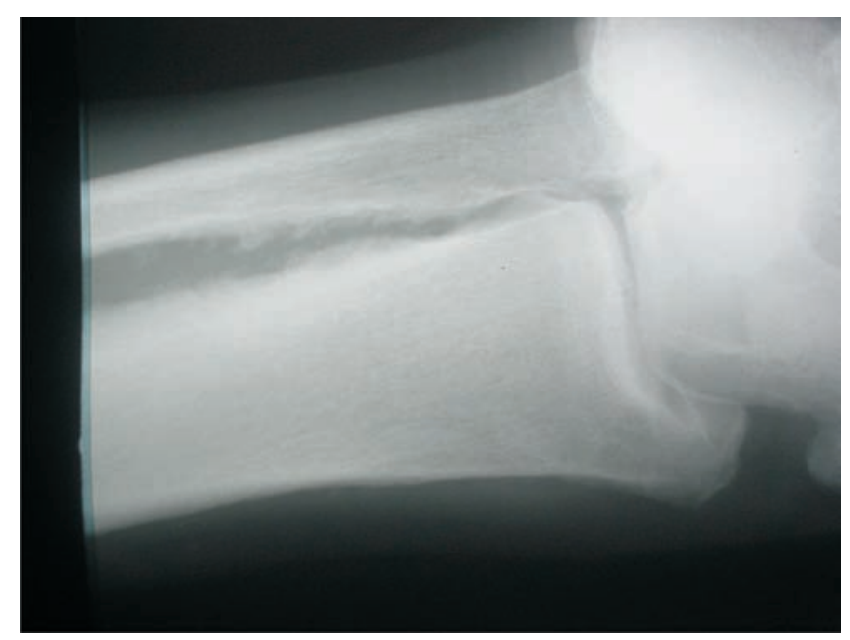

Figura 3 - Radiografia de tornozelo mostrando reação periosteal em ossos da perna.

A citologia global do líquido sinovial foi de 1.600 células $/ \mathrm{mm}^{3}$ com $92 \%$ de linfócitos e $8 \%$ de polimorfonucleares. O fator reumatóide IgM (látex e Waaler-Rose) foi negativo.

O pai e o irmão mais velho de nosso paciente foram convidados a uma consulta para maiores esclarecimentos sobre a história familiar e para nos certificarmos da real presença de OHP nesses indivíduos. Para tanto, procedemos a anamnese e exame físico e ambos apresentavam história de baqueteamento digital iniciada na adolescência (por volta dos 15 anos), sem queixas álgicas associadas. O exame físico, fora o baqueteamento dos dedos das mãos e dos pés, era inespecífico. O estudo radiológico mostrou as mesmas alterações de ossos longos do nosso paciente, sem acometimento de partes moles. Um tio paterno também apresenta baqueteamento, segundo o pai de nosso paciente.

Assim, o nosso paciente S.S.L.S. pôde ser enquadrado no diagnóstico de OHP em virtude de história familiar, início gradual na adolescência e exclusão de outras patologias secundárias que poderiam cursar com osteoartropatia hipertrófica. Podemos também concluir que o tipo de OHP do nosso paciente é a forma incompleta, já que não é portador de Cutis verticis gyrata, mas apresenta paquiperiostose e paquidermia ${ }^{(4)}$.

Para o tratamento, empregamos, inicialmente, indometacina $25 \mathrm{mg}$, duas vezes ao dia, com melhora significativa e recrudescência quando da não-manutenção do tratamento e do uso de álcool. Após revisão da literatura, passamos a 
considerar medicamentos que apresentam menos efeitos colaterais, como colchicina ou bisfosfonatos, com resposta ainda a ser avaliada.

\section{DISCUSSÃO}

A artralgia é relativamente comum na $\mathrm{OHP}$, com ocorrência de aproximadamente $40 \%^{(1,10)}$, chegando a ultrapassar $70 \%$ em alguns relatos ${ }^{(5,11)}$. Geralmente a dor leva o paciente a procurar auxílio médico. Já a artrite é descrita com menor freqüência, variando em $10 \%$ a $30 \%$ dos $\operatorname{casos}^{(7,11)}$. As articulações mais acometidas são dos membros inferiores, principalmente joelhos seguidos por tornozelos ${ }^{(3,5)}$. A razão da intensificação da dor após consumo excessivo de álcool não está claramente definida, apesar de descrita na literatura ${ }^{(18)}$ e vivenciada por nós através de nosso paciente.

Por ser uma doença rara e de evolução limitada, não há grandes descrições na literatura médica sobre o tratamento da artralgia na OHP.

O tratamento convencional inclui o uso de analgésicos, antiinflamatórios não esteroidais, corticóides e anestésicos ${ }^{(3,7,9,16)}$. O uso de bloqueadores do sistema nervoso simpático e a simpatectomia são descritos para melhora apenas da hiperidrose ${ }^{(4,11)}$.

A sinovectomia é uma opção nos casos não responsivos ao tratamento clínico em que há importante acometimento $\operatorname{articular}^{(4,11)}$.

Matucci-Cerinic et al. ${ }^{(19)}$ fizeram uso de colchicina $0,5 \mathrm{mg}$, uma vez ao dia, por uma semana e $0,5 \mathrm{mg}$, duas vezes ao dia, por três semanas em um paciente com OHP que apresentava acrosteólise, artralgia e foliculite recorrente de face. Entre 7 e 15 dias de tratamento, obtiveram melhora dos sinais e sintomas da doença.

Guyot-Douot et al..$^{(3)}$ descreveram o uso de $1 \mathrm{mg} / \mathrm{kg}$ de pamidronato, em dose única, em três pacientes que não

\section{REFERÊNCIAS}

1. Yamamoto-Furusho JK, Rodríguez-Reyna T, Zúñiga J, VargasAlarcón G, Martinez-Lavíin M, Granados J: Immunogenetic study of 3 families with probands of primary hypertrophic osteoarthropathy. Clin Exp Rheumatol 16(5): 629-30, 1998.

2. Bianchi L, Lubrano C, Carrozzo AM, et al.: Pachydermoperiostosis: study of epidermal growth factor and steroid receptors. $\mathrm{Br}$ J Dermatol 132(1): 128-33, 1995.

3. Guyot-Drouot MH, Solau-Gervais E, Cortet B, et al.: Rheumatologic manifestations of pachydermoperiostosis responderam à terapia convencional, já que a ação de tal medicamento em outras doenças osteoarticulares (doença de Paget, mieloma múltiplo, metástases ósseas) é conhecida. Dois pacientes apresentaram grande melhora com redução do consumo diário de analgésicos e o terceiro paciente mostrou melhora moderada. Uma segunda infusão de pamidronato não aumentou a eficácia do tratamento. Os autores sugerem que o bisfosfonato pode ser uma opção quando as demais terapêuticas falharam.

Maeda et al. ${ }^{(20)}$ usaram citrato de tamoxifeno para tratamento de um paciente com OHP que apresentava discreta elevação do nível sérico de estriol, baseados na descrição de aumento do número de receptores de estrogênio nos tecidos afetados de pacientes portadores de $\mathrm{OHP}^{(2)}$. O referido paciente não havia apresentado melhora com tratamento convencional, então se utilizaram $20 \mathrm{~g}$ de citrato de tamoxifeno diariamente. Segundo os autores, houve melhora da dor em poucos dias, sendo a artralgia controlada com o uso do medicamento por cinco meses consecutivos, sem evidências de efeitos colaterais.

Okten et al. ${ }^{(21)}$, baseados nos achados de Maeda et al..$^{(20)}$, utilizaram citrato de tamoxifeno nas mesmas doses anteriores para um paciente com todos os sinais de OHP, dor articular, ginecomastia, mas com níveis séricos de hormônios sexuais normais. Houve melhora completa dos sintomas em duas semanas e redução gradual da ginecomastia. A dose foi reduzida após nove meses e o tratamento, suspenso. Não houve redução do baqueteamento nem das alterações ósseas.

Apesar de benigna e autolimitada, a OHP pode reduzir muito a qualidade de vida do paciente quando acompanhada de dor articular e, mesmo sendo rara, deve ser lembrada quando se depara com baqueteamento digital. Com a pesquisa dos fatores patogênicos da doença, é possível que surjam novas propostas terapêuticas.

preliminary experience with bisphosphonates. J Rheumatol 27(10): 2418-23, 2000.

4. Laredo Filho J, Lazzareschi M: Paquidermoperiostose: atrofia hipertrófica primária: estudo de dois pacientes. Rev Imagem 8(1): 1-8, 1986.

5. Jajic Z, Jajic I, Nemcic T: Primary hypertrophic osteoarthropathy: clinical, radiologic, and scintigraphic characteristics. Arch Med Res 32 (2): 136-42, 2001.

6. Guiringhelli G, Rubin Z, Otaño AS: Paquidermoperiostosis: presentación de um caso. Rev Assoc Argent Ortop Traumatol 55(4): 457-61, 1990. 
7. Cruz MA, Pereira GJC, Pereira HR: Osteoartropatia hipertrófica primária. J Bras Med 69: 145-62, 1995.

8. Irwin RS: Knee discomfort in a 38-year-old man. Chest 122(6): 2242-5, 2002.

9. Favus MJ, Vokes TJ: Harrison medicina interna. 16 ed. Rio de Janeiro: Mc-Graw Hill, 2006.

10. Jajic I, Jajic Z, Grazio S: Minor but important symptoms and signs in primary hypertrophic osteoarthropathy. Clin Exp Rheumatol 19(3): 357-8, 2001.

11. Inocêncio RM, Moreira HNC, Bertolo MB, Samara AM: Osteoartropatia hipertrófica primária. Rev Bras Reumatol 32: 34-40, 1992.

12. Viola IC, Joffe S, Brent LH: Primary hypertrophic osteoarthropathy. J Rheumatol 27(6): 1562-3, 2000.

13. Gaston-Garrette F, Porteau-Cassard L, Marc V, et al.: A case of primary hypertrophic osteoarthropathy without skin involvement (Currarino's disease). Rev Rhum Engl Ed 65(10): 591-3, 1998.

14. Castori M, Sinibaldi L, Mingarelli R, Lachman RS, Rimoin DL, Dallapiccola B: Pachydermoperiostosis: an update. Clin Genet 68(6): 477-86, 2005.
15. Silveira LH, Martinez-Lavin M, Pineda C, Fonseca MC, Navarro C, Nava A: Vascular endothelial growth factor and hypertrophic osteoarthropathy. Clin Exp Rheumatol 18(1): 57-62, 2000.

16. Altman RD, Tenenbaum J: Hypertrophic Osteoarthropathy. In: Kelley's textbook of rheumatology. 7th ed. Philadelphia: Elsevier Science p. 1748, 2005.

17. Oikarinen A, Palatsi R, Kylmaniemi M, Keski-oja J, Risteli J, Kallioinen M: Pachydermoperiostosis: analysis of connective tissue abnormality in one family. J Am Acad Dermatol 31(6): 947-53, 1994.

18. Mueller MN, Trevarthen D: Pachydermoperiostosis: arthropathy aggravated by episodic alcohol abuse. J Rheumatol 8(5): 862-64, 1981.

19. Matucci-Cerinic M, Fattorini L, Lombardi A, et al.: Colchicine treatment in a case of pachydermoperiostosis with acroosteolysis. Rheumatol Int 8(4): 185-8, 1988.

20. Maeda H, Kumagai K, Konishi F, et al.: Successful treatment of arthralgia with tamoxifen citrate in a patient with pachydermoperiostosis. Rheumatology (Oxford) 39(10): 1158-9, 2000.

21. Okten A, Mungan I, Kalyoncu M, Orbak Z: Two cases with pachydermoperiostosis and discussion of tamoxifen citrate treatment for arthralgia. Clin Rheumatol 26(1): 8-11, 2007. 